\title{
DSpace@MIT
}

\author{
MIT Open Access Articles
}

Plasma electron hole kinematics. II. Hole tracking Particle-In-Cell simulation

The MIT Faculty has made this article openly available. Please share how this access benefits you. Your story matters.

Citation: Zhou, C., and I. H. Hutchinson. “Plasma Electron Hole Kinematics. II. Hole Tracking Particle-In-Cell Simulation." Physics of Plasmas 23, 8 (August 2016): 082102

As Published: https://doi.org/10.1063/1.4959871

Publisher: American Institute of Physics (AIP)

Persistent URL: http://hdl.handle.net/1721.1/118621

Version: Final published version: final published article, as it appeared in a journal, conference proceedings, or other formally published context

Terms of Use: Article is made available in accordance with the publisher's policy and may be subject to US copyright law. Please refer to the publisher's site for terms of use. 


\section{Plasma electron hole kinematics. II. Hole tracking Particle-In-Cell simulation}

C. Zhou, and I. H. Hutchinson

Citation: Physics of Plasmas 23, 082102 (2016); doi: 10.1063/1.4959871

View online: https://doi.org/10.1063/1.4959871

View Table of Contents: http://aip.scitation.org/toc/php/23/8

Published by the American Institute of Physics

\section{Articles you may be interested in}

Plasma electron hole kinematics. I. Momentum conservation

Physics of Plasmas 23, 082101 (2016); 10.1063/1.4959870

Electron holes in phase space: What they are and why they matter

Physics of Plasmas 24, 055601 (2017); 10.1063/1.4976854

Electron holes in inhomogeneous magnetic field: Electron heating and electron hole evolution

Physics of Plasmas 23, 052306 (2016); 10.1063/1.4950834

Evolution of electron phase space holes in inhomogeneous plasmas

Physics of Plasmas 24, 062311 (2017); 10.1063/1.4989717

On the nonlinear trapping nature of undamped, coherent structures in collisionless plasmas and its impact on stability

Physics of Plasmas 24, 032109 (2017); 10.1063/1.4978477

Particle dynamics in the electron current layer in collisionless magnetic reconnection

Physics of Plasmas 23, 102102 (2016); 10.1063/1.4963008

\section{PHYSICS TODAY}

MANACER'S GUIDE

WHITEPAPERS

\section{READ NOW}

PRESENTED BY

Accelerate R\&D with Multiphysics Simulation

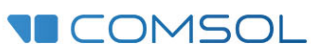




\title{
Plasma electron hole kinematics. II. Hole tracking Particle-In-Cell simulation
}

\author{
C. Zhou and I. H. Hutchinson \\ Plasma Science and Fusion Center, Massachusetts Institute of Technology, Cambridge, Massachusetts 02139, \\ USA
}

(Received 10 May 2016; accepted 13 July 2016; published online 2 August 2016)

\begin{abstract}
The kinematics of a 1-D electron hole is studied using a novel Particle-In-Cell simulation code. A hole tracking technique enables us to follow the trajectory of a fast-moving solitary hole and study quantitatively hole acceleration and coupling to ions. We observe a transient at the initial stage of hole formation when the hole accelerates to several times the cold-ion sound speed. Artificially imposing slow ion speed changes on a fully formed hole causes its velocity to change even when the ion stream speed in the hole frame greatly exceeds the ion thermal speed, so there are no reflected ions. The behavior that we observe in numerical simulations agrees very well with our analytic theory of hole momentum conservation and the effects of "jetting." Published by AIP Publishing.

[http://dx.doi.org/10.1063/1.4959871]
\end{abstract}

\section{INTRODUCTION}

An electron hole is a Bernstein-Greene-Kruskal (BGK) mode $^{1}$ solitary wave which is a self-consistent solution of Vlasov-Poisson system. Other important BGK modes include ion holes and double layers. ${ }^{2}$ These equilibrium solutions were initially predicted by theory and have been observed in space plasmas, laboratory plasma experiments, and computational simulations. ${ }^{3-7}$ Debye-scale bipolar electric field structures typical for electron holes are frequently observed by space probes in earth's magnetosphere and auroral zone of the ionosphere. ${ }^{8-10}$ It has been postulated that these trapped electrons are created during reconnection of earth's magnetic field and play an important role in energization of electrons. ${ }^{11,12}$ Being a collective mode of plasma, an electron hole exhibits a particle-like behavior. Efforts have gone into defining an effective mass, charge, and momentum for these phase-space holes. ${ }^{13}$ Numerical simulations of electron phase-space holes have been an important way to investigate the associated phenomena. Dawson performed a one-dimensional ParticleIn-Cell (PIC) simulation ${ }^{14}$ of a water-bag electron distribution with a hole in it. They observed self-acceleration of the electron phase-space hole in their simulations. They attribute this acceleration to the emission of ion-acoustic waves. Simulations of electron holes in a Vlasov plasma using a continuum code ${ }^{5}$ also show strong acceleration of an electron hole to an important fraction of electron thermal speed after the ion density has formed a deep enough cavity. The interaction between electron hole and surrounding ions has been investigated by Saeki and Rasmussen. ${ }^{15}$ They found by performing particle simulations ${ }^{16}$ that ion motion can lead to the disruption of an electron hole into two holes traveling in opposite directions at several times the ion sound speed.

Electron holes are also present in particle simulation of the wake of an unmagnetized object in flowing magnetized plasma. It is found that electron holes can form arising from unstable electron distribution in the wake. ${ }^{17}$ Some holes will stay in the central wake and grow large enough to disrupt ions while others quickly move out of the wake. ${ }^{18}$

In order to understand the movement of these phasespace holes, we use a hole tracking Particle-In-Cell simulation code which is one-dimensional electrostatic. It gives a good representation of electron holes in the presence of a relatively strong magnetic field such as in the earth's magnetosphere. We have fully kinetic ions in our simulation, as the effect of ion dynamics on electron holes is what we are interested in. Simulation of a single solitary electron hole is performed in a modest sized box with open boundary conditions rather than periodic. Electrons and ions have fixed Maxwellian distributions outside the boundaries.

This paper is organized in the following way: the hole tracking particle simulation implementation is described in detail in Section II. Section III presents the results from hole tracking PIC simulation of electron holes in initial transient and steady state motion. Observations from simulations are compared with an analytic theory. Section IV is devoted to numerical experiments where we artificially accelerate ion streams to push or pull the hole. They show important momentum coupling between electron hole and ions. Final summary comments are given in Section V.

\section{HOLE TRACKING SIMULATION}

Periodic boundary conditions are avoided in this study because the hole can interact with itself through the periodic boundaries, masking the phenomena we are studying. Open boundary conditions for particles are used in our simulation. Particles are free to leave the computation domain and new particles are injected at every time step to represent the Maxwellian distribution of ions and electrons in surrounding plasma. Boundary conditions for potential $\phi$ are homogeneous with $\phi^{\prime}(x)=\phi(x) / \lambda_{\text {De }}$ and $\phi^{\prime}(x)=-\phi(x) / \lambda_{\text {De }}$ at the two ends of our simulation domain. These boundary conditions assume that electrostatic potential falls exponentially to zero on the Debye length scale in surrounding plasma. Electron holes move at several times the cold ion sound speed up to electron thermal speed ${ }^{2}$ relative to bulk species, while the spatial extent of an electron hole is only a couple of Debye lengths. The total distance traveled by an electron hole for a long run is therefore hundreds or thousands of its own size. A non-periodic simulation of the long term evolution of an electron hole would require thousands of Debye 
lengths in domain size if a fixed domain is used. An electron hole is sensitive to the statistical noise level in PIC simulation. For $N_{s}$ particles per computation cell, the statistical noise level on charge density scales like $1 / \sqrt{N_{s}} \cdot{ }^{19}$ To have a clear resolution of the hole, we need a large number of particles per Debye length. The combination of a large simulation domain with the requirement of a high number of particles per length would demand excessive computational resources. Simulating a fast moving electron hole using a fixed domain is not efficient.

Therefore, we adopt a more adaptive approach to this problem. A 1-D Particle-In-Cell (PIC) code is used which detects the hole signal and moves the computation domain accordingly so that the hole always stays inside it. We refer to this method as "hole tracking." The major component of hole tracking is a feedback control mechanism. The hole position is detected with an automated search algorithm. At every time step, electric field from the simulation is convolved with a bipolar hole electric field signal. The hole potential in its moving frame is considered to follow approximately the analytic solution ${ }^{20}$

$$
\phi(x)=\psi \operatorname{sech}^{4}\left(\frac{x}{L}\right)
$$

which gives rise to a bipolar electric field of the form $E(x)=-\frac{4 \psi}{L} \tanh \left(\frac{x}{L}\right) \operatorname{sech}^{4}\left(\frac{x}{L}\right)$. The typical hole half width $L$ is taken to be $4 \lambda_{\mathrm{De}}$. This choice will be justified later in Section III. However, we observe that the choice of $L$ affects very little hole tracking results as long as it is in the vicinity of a few Debye lengths. Position of the filtered signal maximum is taken to be where the electron hole is. Hole search algorithm for on board solitary wave detection ${ }^{21}$ of space probes is generally more sophisticated than the one presented here. However, our algorithm achieves good performance for the purpose of this study and is easy to implement.

Figure 1 shows a block diagram of the code's major components. At every time step $k$, the hole search algorithm gives the position of electron hole $x_{h}[k]$ relative to the simulation domain. Lab frame hole velocity $v_{h}[k]$ is given by

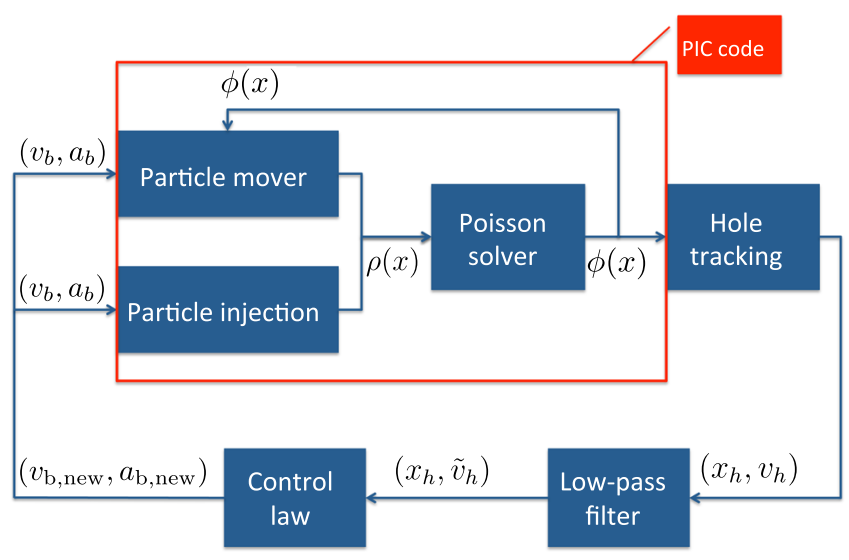

FIG. 1. Block diagram of hole tracking, $\rho(x)$ and $\phi(x)$ are charge density and potential in the simulation, $x_{h}$ and $v_{h}$ are position and velocity of electron hole, $\tilde{v}_{h}$ is the hole velocity after smoothing is applied, $v_{b}$ and $a_{b}$ are velocity and acceleration of simulation box. $\left(x_{h}[k]-x_{h}[k-1]\right) / \delta t+v_{b}[k]$, where $\delta t$ is the time step size of simulation and $v_{b}[k]$ is the velocity of simulation domain from time step $k-1$ to $k$. The hole speed calculated this way is subject to high frequency noise. There is statistical noise that is intrinsic to PIC simulation and sampling noise reflecting the fact that hole position can only be evaluated with a finite precision limited by search algorithm spatial grid step. The noise is amplified by taking the numerical time derivative. Consequently, a low-pass filter is required to filter out high frequency noise in measured hole velocity. We adopt a causal Butterworth filter of second order in our simulation. The cutoff frequency is empirically chosen as $0.005 \omega_{p e}$. The higher the cutoff frequency, the faster the control law will respond to changes in the hole velocity but it will also make it more sensitive to noise. The filtered hole velocity $\tilde{v}_{h}[k]$ is used as an input of control law to extrapolate the simulation box velocity $v_{b}[k+1]$ that is required to follow the hole motion. The control law takes both hole velocity $\tilde{v}_{h}[k]$ and relative position $x_{h}[k]$ as input for robust feedback control on both velocity and position. The control law can be expressed as

$$
\frac{v_{b}[k+1]-v_{b}[k]}{\delta t}=K_{1} x_{h}[k]+K_{2}\left(\tilde{v}_{h}[k]-v_{b}[k]\right),
$$

where $K_{1}$ and $K_{2}$ are, respectively, control coefficients on position and velocity.

There is a certain freedom in the choice of control coefficients $K_{1}$ and $K_{2}$. The control law should respond fast enough so that the electron hole does not leave the domain but not induce instability or excessive overshoot. In our simulation, position is normalized to $\lambda_{\mathrm{De}}$ and velocity is normalized to $c_{\mathrm{S}}=\sqrt{\frac{T_{e}}{m_{i}}}$. The control coefficients we adopt are then $K_{1}=0.0025 \omega_{p e}^{2}$ and $K_{2}=0.75 \omega_{p e}$.

The particle pushing and particle injection parts of the PIC code need to take into account the fact that the simulation domain is moving relative to the background plasma and is accelerating. As a consequence of acceleration, the simulation domain is no more an inertial frame of reference. In addition to the force due to the electric field, particles feel an extra acceleration which is the opposite of box acceleration $a_{b}$. This term is included during particle pushing. Background plasma particle distribution relative to simulation domain is now a Maxwellian shifted by minus the box velocity $-v_{b}$. The velocity distribution of particle fluence across simulation boundaries can be written as

$$
|v| f(v) d v=\frac{n_{0}|v|}{\sqrt{2 \pi} v_{\text {th }}} \exp \left[-\frac{\left(v+v_{b}\right)^{2}}{2 v_{\text {th }}^{2}}\right] d v,
$$

where $v_{\text {th }}=\sqrt{\frac{T_{i / e}}{m_{i / e}}}$. Eq. (3) can be integrated to obtain the total number of particles that need to be injected into the simulation box during a time step of length $\delta t$

$$
N_{\text {injection }}=\frac{2 n_{0} v_{\mathrm{th}} \delta t}{\sqrt{2 \pi}} \exp \left(-\frac{v_{b}^{2}}{2 v_{\mathrm{th}}^{2}}\right)+n_{0} v_{b} \delta t \operatorname{erf}\left(\frac{v_{b}}{\sqrt{2} v_{\mathrm{th}}}\right) \text {. }
$$

At each iteration, $N_{\text {injection }}$ particles are injected following a distribution given by Eq. (3) for both ions and electrons. The 
velocity sign of a particle determines which boundary it will be injected from. $N_{\text {injection }}$ and Eq. (3) need to be evaluated at every iteration as $v_{b}$ is constantly changing as a result of feedback control. A standard acceptance-rejection method ${ }^{22}$ is implemented to draw random velocities from a distribution expressed by Eq. (3). The particle injection is considered to be uniform in time. Once injected, they will experience a partial kick and drift. The time duration of this partial particle push is $\theta \delta t$, where $\theta$ is a random number uniformly distributed between 0 and 1 . The PIC simulation uses a leapfrog integration scheme. As a consequence, velocities of particles are always at half a time step behind their positions. Injection is made consistent with this leapfrog scheme, otherwise unphysical density perturbations are excited at the simulation boundaries. The standard PIC component of the code is a 1-D electrostatic Particle-In-Cell code, which is referred to as ESPIC. Charge weighting in the code uses a cloud-incell ${ }^{19}$ approach and Poisson equation is solved by a direct tridiagonal method. A detailed description of ESPIC can be found in a previous publication ${ }^{18}$ by Haakonsen et al.

We choose the length of simulation domain to be $48 \lambda_{\mathrm{De}}$ across. By virtue of hole tracking, we do not need a very big domain size to simulate a fast moving hole. However, it should be large enough so that electron holes stay away from boundaries during simulations. In this way, unphysical boundary effects can be avoided. The longest excursion that a hole makes from the center of simulation box is around $15 \lambda_{\mathrm{De}}$ before it is caught up by hole tracking in all of our simulations. This choice of domain size guarantees a safety margin from boundaries without being too demanding on computational resources. In order to seed a phase-space hole, we initialize the electron distribution with a phase-space perturbation. The details of this initialization will be discussed in Section III. Hole tracking can be considered as a technique to simulate an electron hole in its rest frame. A successful hole tracking simulation should not change the physics we are trying to simulate.

Figure 2 compares velocity evolution of an electron hole in an ordinary fixed domain PIC run to what we obtain from a hole tracking PIC run using the same initialization setting. The fixed domain run uses a domain which is $192 \lambda_{\text {De }}$ across

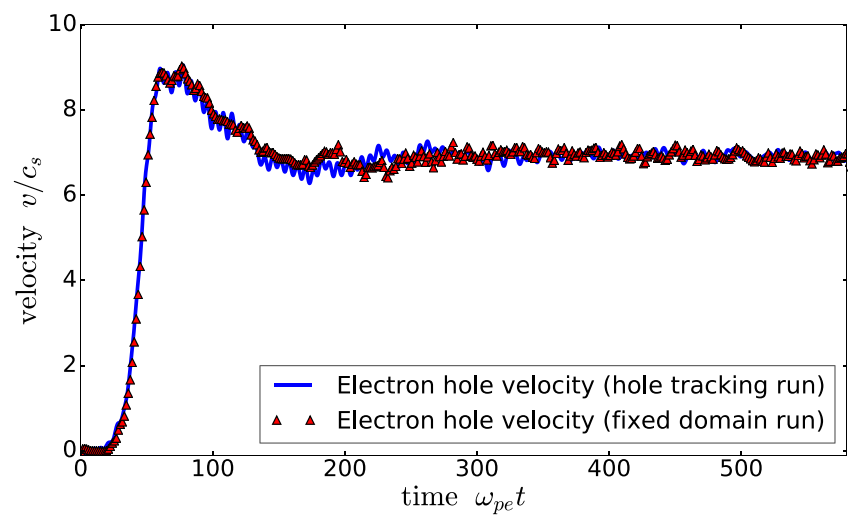

FIG. 2. Velocity of an electron hole in two different runs, in the fixed domain run, the electron hole hits the boundary at $\omega_{\text {pe }} t=590$ while the hole tracking run can successfully simulate hole motion for a much longer period of time. Velocity data shown here are smoothed using a low-pass filter of cutoff frequency $0.15 \omega_{p e}$. with $2 \times 10^{8}$ particles and 4000 spatial cells. The hole tracking run uses a standard $48 \lambda_{\text {De }}$ domain and thus only requires a quarter as many particles and spatial cells. The agreement between the two runs demonstrates the strength of hole tracking, which is able to resolve hole motion to the same precision with less computational resources. And for longer time durations, the gain would be even greater.

We use $10^{7}-10^{9}$ particles in our simulations depending on the size of hole we want to simulate. Simulating a shallower hole requires more particles to keep the same signalto-noise ratio. The number of cells is chosen to be $10^{3}$ so that we have $\sim 20$ grid-points per Debye length, making sure that the hole structure is well resolved. The code is fully parallelized to meet the challenge of the large number of particles and time steps required to resolve the phenomena we are interested in. It is observed that a choice of time step size bigger than $0.5 / \omega_{p e}$ may drive the simulation numerically unstable. We have explored a range of different time step sizes in our simulation from $0.01 / \omega_{p e}$ to $0.4 / \omega_{p e}$. The choice of time step size does not affect simulation results if it is below the stability threshold. $\delta t=0.3 / \omega_{p e}$ is the choice we adopt in our simulations.

Feature tracking PIC simulation is a very versatile tool by its nature. A similar approach can be used to investigate the highly resolved dynamics of a wide class of nonlinear plasma phenomena, such as the formation and kinematics of ion holes.

\section{INITIAL TRANSIENT TO STEADY STATE}

There are different ways to seed a phase-space hole at the initial stage of a numerical simulation. Schamel derived an analytic solution for electron hole structure in the absence of ion response. ${ }^{20}$ This analytic expression of electron distribution function has been used as initialization of hole simulation by Eliasson and Shukla. ${ }^{5}$ However, we adopt a different approach in our simulation which can be divided into the following steps:

Step 1: For a given electron density $n_{0}$, each electron is initialized with a random position which is uniformly distributed in spatial domain. A Quiet Start ${ }^{19}$ technique is used for position initialization to make sure that the number of particles in each spatial cell is uniform.

Step 2: For a given electron thermal velocity $v_{\text {th,e }}=\sqrt{\frac{T_{e}}{m_{e}}}$, each electron is initialized with a random velocity according to a probability distribution which is a Maxwellian

$$
\tilde{f}_{e, 0}=\frac{1}{\sqrt{2 \pi} v_{\mathrm{th}, \mathrm{e}}} \exp \left(-\frac{v^{2}}{2 v_{\mathrm{th}, \mathrm{e}}^{2}}\right) .
$$

Step 3: For each electron with a position $x$ and a velocity $v$, a random number $\alpha$ is drawn uniformly between 0 and 1 , the velocity is rejected if $\alpha$ is smaller than a predefined value $f_{d}(x, v)$, where

$$
f_{d}(x, v)=h_{d} \exp \left(\frac{-\left(v-v_{d}\right)^{2}}{2 \sigma_{d}^{2}}\right) \exp \left(\frac{-x^{2}}{2 \lambda_{d}^{2}}\right)
$$


This is a bell-shaped function in space and velocity: $h_{d}$ is the dimple depth which is smaller than $1 ; \sigma_{d}$ is the dimple velocity width; $v_{d}$ is the dimple initial velocity; and $\lambda_{d}$ is the dimple spatial width.

If a velocity is rejected in step 3, we return to step 2 and choose a new velocity and then move on to step 3 to go through another rejection test with this new velocity, iterating till the velocity is accepted. By this process, we initialize a dimple in the velocity distribution, localized in position and velocity, but maintain the initial density uniform by enhancing the rest of the electron distribution. Since at each rejection step, the same fraction of velocities (determined by $f_{d}$ ) is rejected, the final distribution is proportional to $\tilde{f_{e 0}}\left(1-f_{d}\right)$. And since the total density is uniform, the normalization gives

$$
f_{e, 0}=n_{0} \frac{\tilde{f}_{e, 0}-\tilde{f}_{e, 0} f_{d}}{1-\int_{-\infty}^{+\infty} \tilde{f}_{e, 0} f_{d} d v},
$$

$f_{e, 0}$ is the initial electron distribution in our simulation. This hole seeding process is simple to implement but not exactly self-consistent. However, the initial phase-space perturbation will evolve into a self-consistent hole. We refer to the process described above as "uniform density initialization." The uniform density initialization reduces the initial plasma oscillations in the simulation due to charge imbalance.

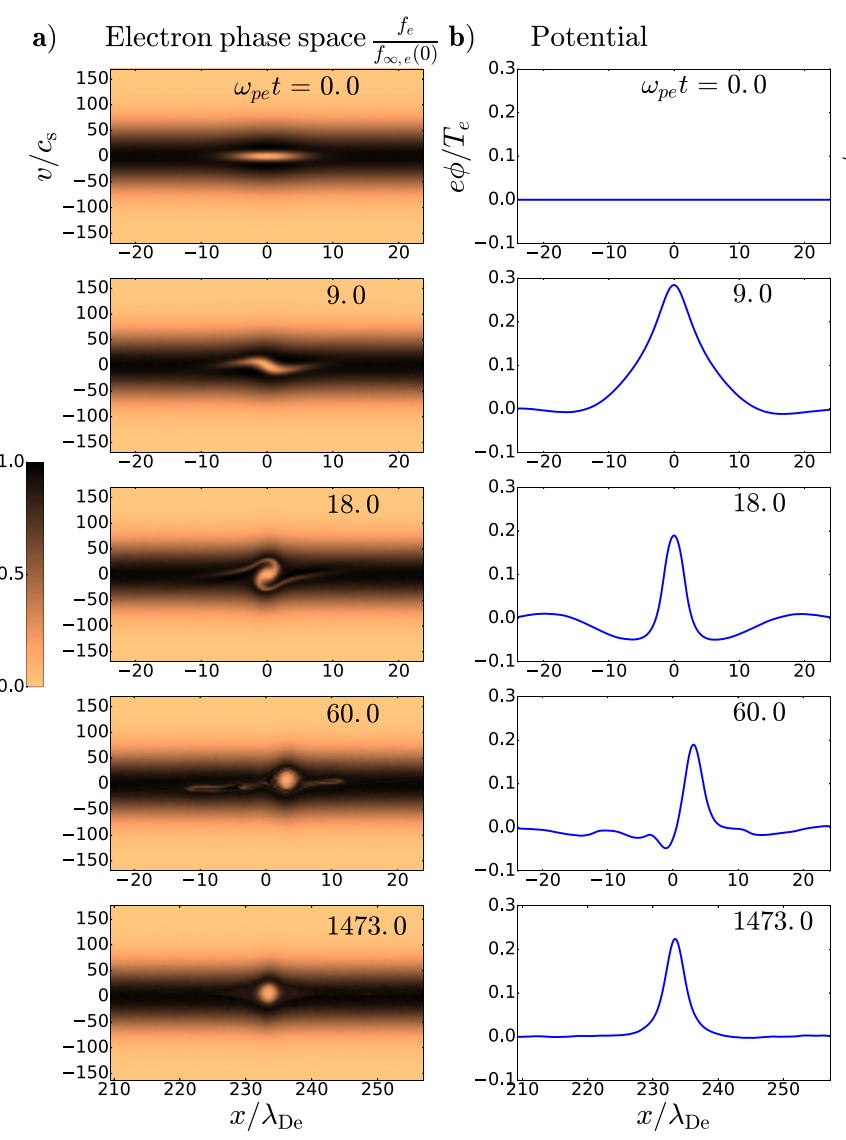

Figure 3 shows a run with uniform density initialization and the subsequent transient. The ions are initialized and injected as a Maxwellian distribution with a temperature $T_{i}$ and a drift velocity of $-1 c_{s}$ in lab frame. The temperature ratio $T_{e} / T_{i}$ is 20 , mass ratio $m_{i} / m_{e}=1836$. The ions are beam like in this simulation. Initialization parameters for electron phase-space density deficit are $h_{d}=0.9, v_{d}=0, \sigma_{d}$ $=0.15 v_{\text {th.e }}, \lambda_{d}=4 \lambda_{\mathrm{De}}$. The number of particles used in this run is $N_{e}=N_{i}=5.12 \times 10^{7}$.

The first row of Figure 3 shows the initialization of simulation, notice the initial dimple shape of deficit in electron phase-space density. Ion and electron densities are equal and uniform by virtue of Quiet Start. As a consequence, the initial potential is zero for our initialization. Once simulation starts, the 2nd and 3rd rows of column (a) in Figure 3 show that the initial perturbation in electron phase-space begins to rotate, following the phase-space flow. Its aspect ratio changes during this process. The same rows of column (d) show that a cavity quickly appears in electron density, giving rise to a positive potential pulse which in turn traps the low energy electrons inside. Row 4 shows that after ions have time to respond to this potential $\left(\omega_{\text {pe }} t>\left(m_{i} / m_{e}\right)^{\frac{1}{2}}\right)$, a cavity of depth $\simeq 5 \%$ forms in ion density at the initial position of the hole. The initial ion density perturbation is deeper for a smaller mass ratio $\left(\simeq 13 \%\right.$ for $\left.m_{i} / m_{e}=100\right)$. The electron hole is ejected by this ion density cavity, speeding up during this process. Ions are initialized with a negative drift velocity in the lab frame so that the electron hole is ejected in the

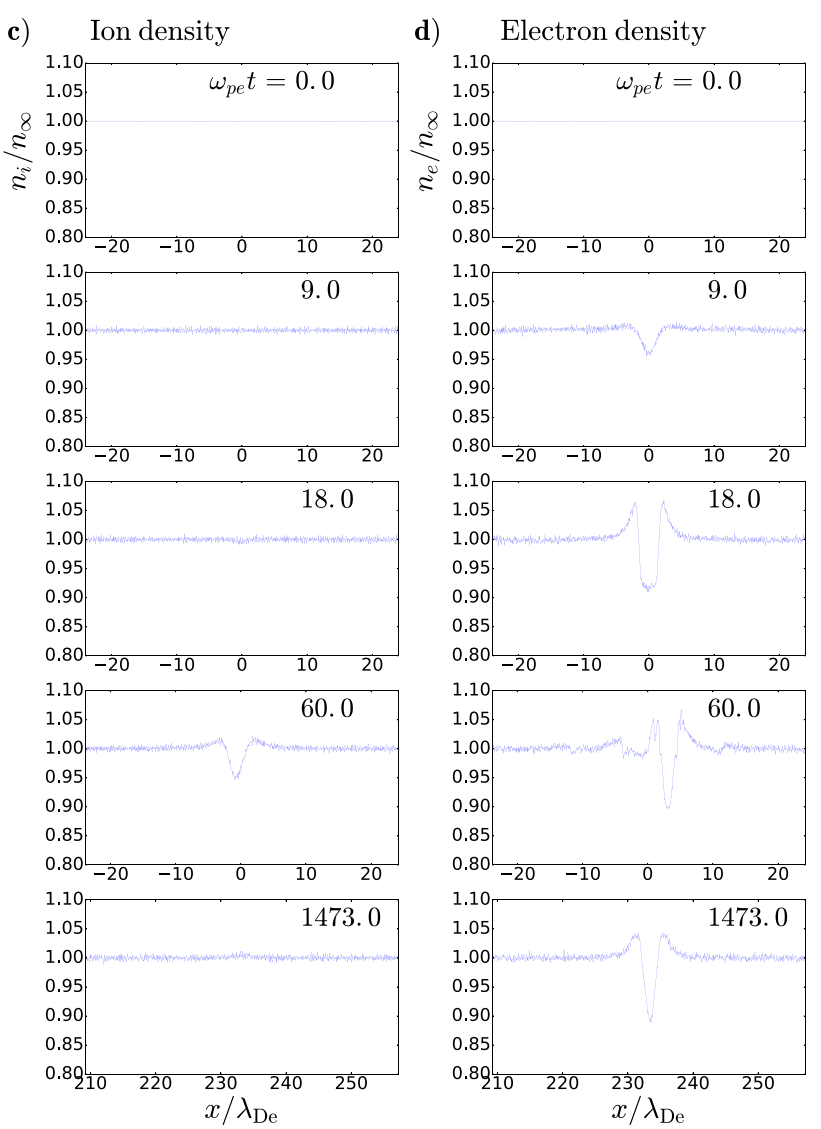

FIG. 3. (a) Normalized electron phase-space density contours, (b) potential, (c) ion density, and (d) electron density. Position $x$ and velocity $v$ are relative to lab frame. The plots shown on the same row are from the same time step in the simulation. 
positive $x$ direction in this run. Once ejected, the electron hole leaves the ion perturbation and other transient remnants behind it and moves into uniform background plasma. The fully formed self-consistent hole has a shorter spatial width than initialization in this case.

The ion density perturbation eventually moves out of the simulation domain and our simulation tracks the hole into background plasma. The $x$ axis labels in Figure 3 give the absolute position in lab frame. The last row shows that the electron hole has traveled more than $200 \lambda_{\text {De }}$ but is still well-centered in our simulation domain, thanks to hole tracking. A steady state hole can be observed in its rest frame. The potential height of the fully formed electron hole $e \psi / T_{e}$ is approximately 0.23 for this run, where $\psi$ is the maximum of hole potential. The attached ion-acoustic soliton structure described by Saeki and Genma ${ }^{16}$ is not very visible in the steady state hole because the ions are all traveling at $\simeq 8 C_{S}$ in the hole frame. The average ion kinetic energy in the hole frame is much bigger than $e \psi$. The perturbation in the ion density due to the hole is therefore negligible. The electron density has a deep cavity with excess of electrons around it due to shielding, which is typical of an electron hole. ${ }^{2}$

The lab frame velocity of electron hole and simulation domain are plotted in Figure 4 for this same run. The feedback control law expressed by Eq. (2) has both proportional and integral terms of velocity error. As a consequence, both differences in velocity and position between electron hole and simulation domain eventually vanish. We can observe the detailed evolution of electron hole dynamics in Figure 4. At the beginning, there is an initial dwell in the hole velocity. It corresponds approximately to the growth time of initial ion density perturbation. Then the electron hole is rapidly accelerated and its velocity reaches $8.9 c_{s}$ at $\omega_{p e} t=60$, this corresponds to the time it leaves the initial ion density cavity. Once moving into uniform background plasma which has a higher ion density, the electron hole is decelerated and its velocity finally stabilizes around $6.9 c_{s}$ at $\omega_{p e} t \approx 130$.

An important question arises, what determines the final steady state velocity of an electron hole? To answer this question, we have performed a detailed quantitative study of

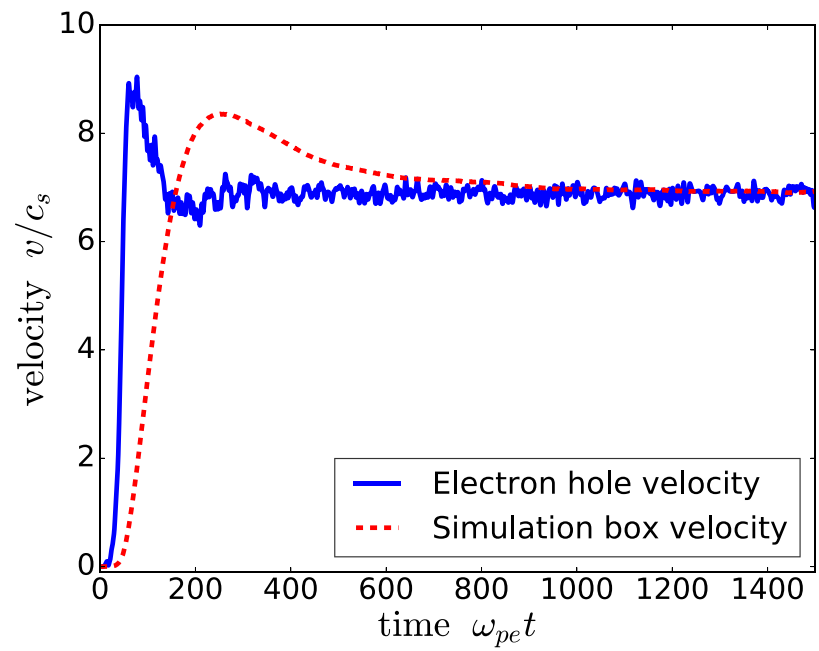

this transient acceleration. Fifty runs have been carried out using hole tracking PIC for different ion to electron mass ratios $m_{i} / m_{e}$, hole depths $\psi$, and initial ion drift velocity $v_{i}$. We take advantage of the flexibility of our code to explore a wide range of parameters. Mass ratio can be easily changed in our simulation. Hole depth is controlled by parameters of uniform density initialization described at the beginning of this section. A deeper dimple depth $h_{d}$ combined with a bigger dimple velocity width $\sigma_{d}$ will give rise to a deeper and wider deficit in initial electron phase-space density. It will then evolve into a deeper self-consistent hole. The dimple spatial width $\lambda_{d}$ is kept constant at $4 \lambda_{\text {De }}$. We shall see that the spatial widths of electron holes in our simulation are close to $4 \lambda_{\mathrm{De}}$, despite difference in their depths. A choice of $\lambda_{d}$ which is too wide can give rise to multiple holes. We also use $v_{d}=0$ for initialization, which means the initial electron phase-space density deficit has zero average velocity in lab frame. Electrons are initialized and injected with zero drift velocity in lab frame, ions are initialized and injected with a drift velocity $v_{i}$ in lab frame. This initial drift velocity between ions and electron hole is very important to hole dynamics. Electron to ion temperature ratio $T_{e} / T_{i}$ is 20 . We have $v_{i}<v_{\text {th,e }}$ for all our runs so that Buneman type of instability is avoided. ${ }^{23}$ Ion-electron acoustic type of instability in principle can be excited for some of these runs but it has a very weak growth rate ${ }^{24}$ and is not observed in our simulation. No disruptive plasma instabilities are observed in the runs presented and hole tracking works properly. Each run consists of a hole tracking simulation of 10000 time steps with a step size of $0.3 / \omega_{p e}$. All of the runs except for two use $N_{i}=N_{e}=2.56 \times 10^{7}$ as the total number of particles. It becomes more and more computationally challenging as we try to push the runs to shallow hole limit. The two shallowest holes we have explored require $10^{9}$ particles for successful hole tracking.

Each run is examined individually to determine when exactly the hole enters steady state motion. Steady state quantities such as hole depth and hole velocity are calculated by taking their average value over 1000 time steps right after the steady state is reached. Figure 5 gathers the results from

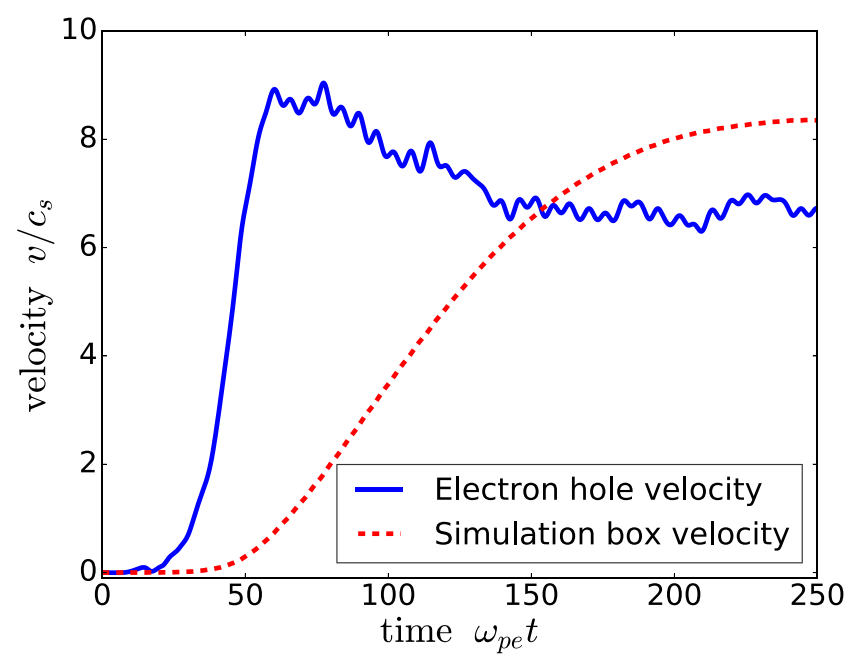

FIG. 4. Lab frame velocity of electron hole and simulation domain, the plot on the right is a close-up examination of the initial transient for the same run, hole velocity is smoothed using a low-pass filter of cutoff frequency $0.15 \omega_{p e}$. 

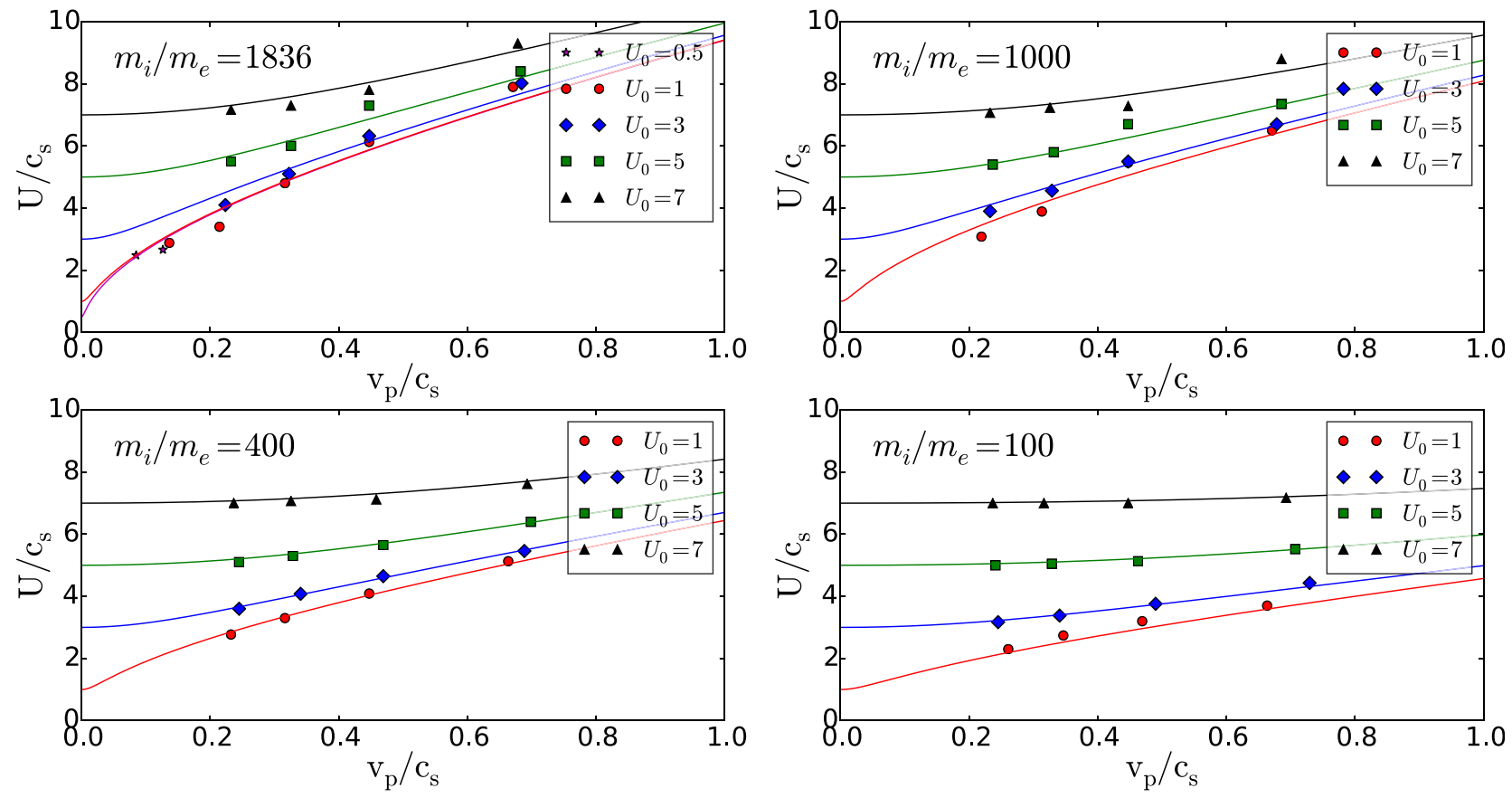

FIG. 5. Steady state hole velocity in initial ion rest frame as a function of mass ratio, steady state hole depth and initial hole velocity, solid curves in each plot are obtained from hole momentum conservation theory.

these runs we have done using different parameters. $U$ is the velocity of an electron hole in the initial rest frame of ions. Therefore, we have $U=v_{h}-v_{i}$ with our initialization process and its initial value $U_{0}$ is equal to $-v_{i} . v_{p} / c_{s}$ $=\sqrt{2 e \psi / T_{e}}$ is the normalized ion passing velocity at hole center, which is proportional to the square root of hole depth.

Our hole momentum conservation theory ${ }^{25}$ predicts the hole kinematics. The theory assumes no specific hole structure and can be applied to electron holes in our simulation. It is our goal here to compare simulation results with our analytic theory. For an electron hole generated from uniform density initialization, the theory provides an explanation for the initial hole acceleration. The change in ion momentum due to hole potential growth must be balanced by change in hole velocity. It can be shown that this initial transient acceleration is governed by the following differential equation derived from hole momentum balance: ${ }^{25}$

$$
-\frac{m_{e}}{m_{i}} h\left(\frac{1}{\sqrt{2}} \frac{v_{p}}{c_{s}}\right) \dot{U}-\frac{3}{4} \frac{v_{p}^{4}}{U^{4}} \dot{U}+\frac{v_{p}^{3}}{U^{3}} \dot{v}_{p}=0,
$$

where the function $h$ for holes that move slowly compared with electron thermal speed $\left(v_{h} \ll v_{\text {th,e }}\right)$ is

$$
h(\chi)=-\frac{2}{\sqrt{\pi}} \chi+\left[\left(2 \chi^{2}-1\right) e^{\chi^{2}} \operatorname{erfc}(\chi)+1\right] .
$$

Function $h$ comes from the expression of electron momentum rate of change when an electron hole is present in electron distribution function. $v_{p}=\sqrt{2 e \varphi / m_{i}}$ the ion passing velocity is a function of the growing potential $\varphi(t)$. The initial conditions are $U(t=0)=U_{0}$ and $\varphi(t=0)=0$. Thus, we have $U\left(v_{p}=0\right)=U_{0}$. Eq. (8) can then be solved for $U$ as a function of $v_{p}$ using a standard Runge-Kutta scheme for different initial values of $U_{0}$ and mass ratio $m_{i} / m_{e}$.
Solutions are plotted as solid lines in Figure 5. The theory predicts that the velocity of an electron hole in ion rest frame follows $U\left(v_{p}\right)$ curve when its depth grows. In the lab frame, this would mean that uniform density initialization with an ion drift velocity $-U_{0}$ gives rise to a steady state hole of velocity $U\left(v_{p}\right)-U_{0}$ when its potential grows from 0 to $e \psi=\frac{1}{2} m_{i} v_{p}^{2}$. Figure 5 shows quite good agreement between simulation results and theoretical $U\left(v_{p}\right)$ solutions. In terms of change in hole velocity $\Delta U=U\left(v_{p}\right)-U_{0}$, the quantitative agreement between theory and simulations is within $20 \%$. The cases where the prediction of analytic theory deviates from simulation results are the ones with small $U_{0}$. Our theory assumes a short-transit-time approximation for ions. The transit time of ions through hole region needs to be much shorter than the typical acceleration timescale $U / \dot{U}{ }^{25}$ This approximation is barely adequate when the ion velocity is slow relative to the hole and important initial acceleration occurs, which corresponds to the runs with small $U_{0}$.

The steady state hole we obtain in our simulation is a very stable coherent structure whose amplitude hardly decays over thousands of electron plasma periods if the noise level in the simulation is kept low by using a sufficient number of particles. Schamel derived an analytic solution ${ }^{20}$ for the shape of a slowly moving $\left(v_{h} \ll v_{\text {th,e }}\right)$ steady state electron hole in the limit of small amplitudes $\left(e \psi / T_{e} \ll 1\right)$. Electron holes in our simulations generally satisfy these two conditions. The form of this solution is given in Eq. (1). The hole half width $L$ is given by the nonlinear dispersion relation ${ }^{2}$

$$
L=\left(-\frac{32}{Z_{\mathrm{Re}}^{\prime}\left(v_{h} / \sqrt{2} v_{\mathrm{th}, \mathrm{e}}\right)}\right)^{\frac{1}{2}} \lambda_{\mathrm{De}}
$$

$Z_{\mathrm{Re}}$ is the real part of plasma dispersion function. ${ }^{26}$ For $|x| \ll 1$, 

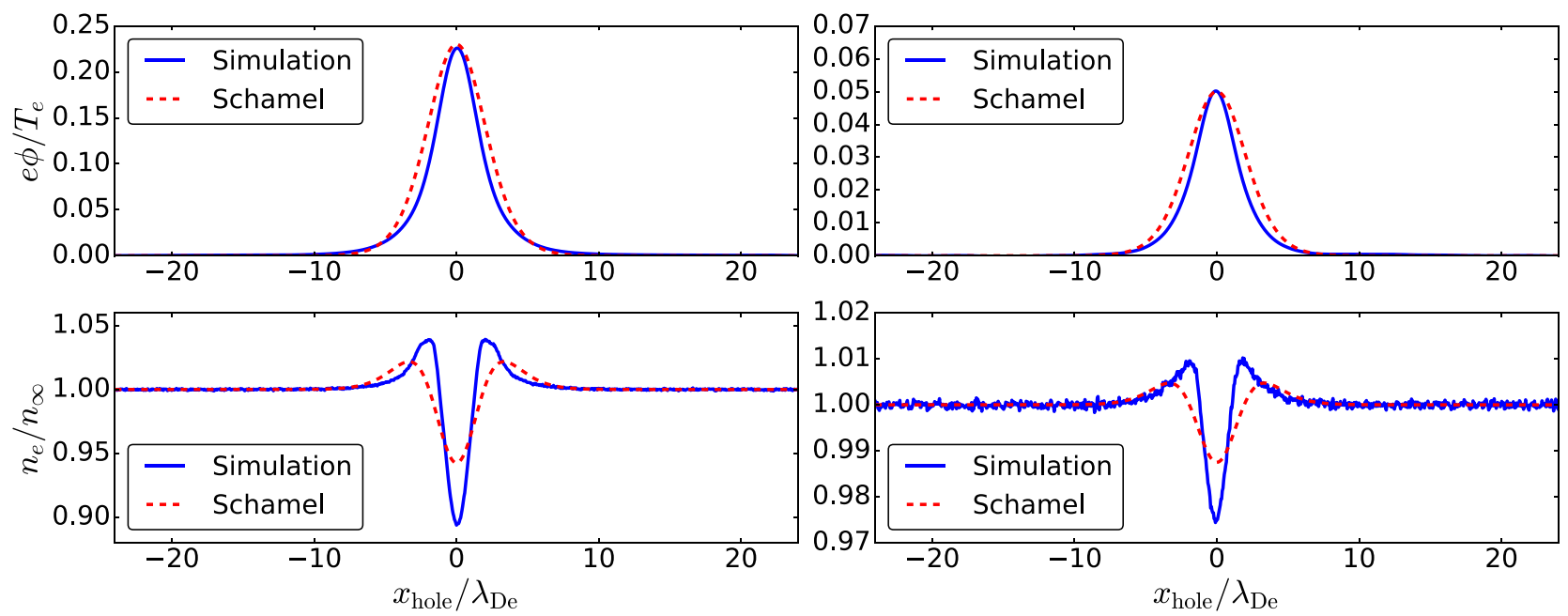

FIG. 6. Potential and electron density profile for two steady state electron holes of different depths and speed compared with Schamel's analytic solution, $e \psi=0.23 T_{e}, v_{h}=6.9 c_{s}$ on the left and $e \psi=0.05 T_{e}, v_{h}=3.8 c_{s}$ on the right, simulation results are averaged over 100 time steps to reduce fluctuations.

$$
Z_{\mathrm{Re}}(x) \approx-2 x\left[1-\frac{2}{3} x^{2}+\frac{4}{15} x^{4}+\ldots\right] .
$$

These expressions yield $L \rightarrow 4 \lambda_{\text {De }}$ when $v_{h} / v_{\text {th,e }} \rightarrow 0$. For $v_{h}=6.9 c_{s}, L \approx 4.05 \lambda_{\text {De }}$ by evaluating Eq. (10). Schamel's analytic expression and the steady state holes in our simulation are plotted in Figure 6.

Analytic curves are generated with $L=4 \lambda_{\text {De }}$ and the maximum of potential measured in the simulation is taken as $\psi$. The analytic expression for electron density is derived by taking the second derivative of Schamel's expression for hole potential. The steady state holes in our simulations are slightly narrower than Schamel's analytic form and also have a deeper dip in electron density. Nevertheless, $L \simeq 4 \lambda_{\text {De }}$ holds for holes of different depths and using Schamel's form in hole search algorithm is an acceptable approximation and yields good performance. For the hole tracking simulation, we can find the electron distribution in the rest frame of an electron hole. The velocity and position of an electron in the simulation domain are the same as their values in the hole frame when the hole is in steady-state motion. We compare the distribution from our simulation with Schamel's solution. Figure 7 shows the value of hole-frame electron distribution function $f_{e}$ as a function of total energy, $\mathcal{E}=\frac{1}{2} m_{e} v^{2}-e \phi(x)$, normalized to its separatrix value $f_{s}=f_{\infty, e}(0) \exp \left(-v_{h}^{2} / 2 v_{\mathrm{th}, \mathrm{e}}^{2}\right)$.

Electrons that have negative energy in hole frame are trapped and those with positive energy are passing. Schamel's solution ${ }^{20}$ is given by

$f_{e}(x, v)= \begin{cases}f_{\infty, \mathrm{e}}(0) \exp \left[-\frac{m_{e}\left(\sigma \sqrt{2 \mathcal{E} / m_{e}}+v_{h}\right)^{2}}{2 T_{e}}\right] & \text { if } \mathcal{E}>0 \\ f_{\infty, \mathrm{e}}(0) \exp \left(-\frac{v_{h}^{2}}{2 v_{\mathrm{th}, \mathrm{e}}^{2}}\right) \exp \left(-\frac{\beta \mathcal{E}}{T_{e}}\right) & \text { if } \mathcal{E}<0,\end{cases}$

where $\sigma=\operatorname{sign} v$ and $\beta$ is the trapped particle parameter. It can be shown that for a slowly moving shallow hole, ${ }^{2} \beta$ is given by
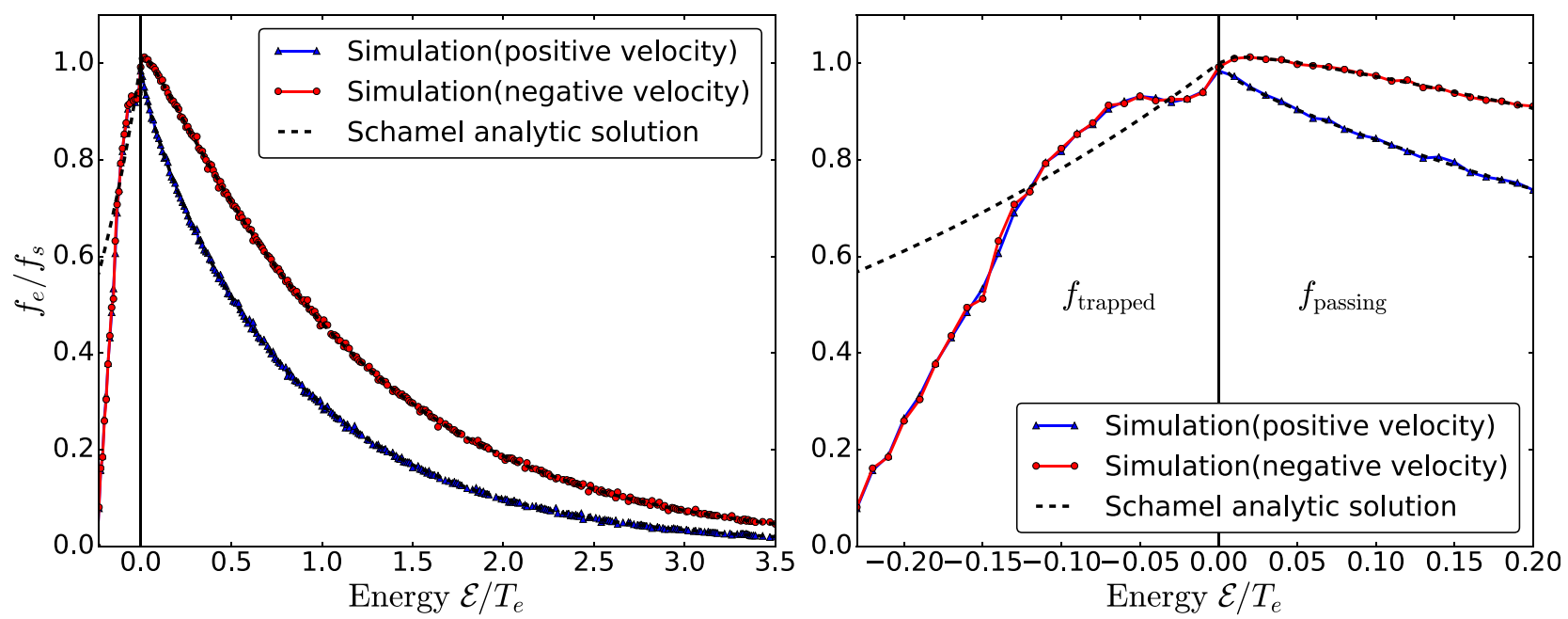

FIG. 7. Electron distribution on constant energy $\mathcal{E}$ orbit in hole frame, $e \psi=0.23 T_{e}, v_{h}=6.9 c_{s}$. Dashed line is Schamel's solution for an electron hole with the same depth traveling at the same speed relative to bulk electrons. 


$$
\beta \simeq 1-\frac{15}{16} \pi^{1 / 2}\left(e \psi / T_{e}\right)^{-1 / 2} .
$$

For $e \psi=0.23 T_{e}$, the value of $\beta$ is -2.47 .

Schamel's solution coincides with a Maxwellian distribution shifted by minus the hole velocity $-v_{h}$ for passing electrons. In the trapped region, Schamel's solution is Maxwell-Boltzmann shaped. The distribution obtained from our simulation agrees with Schamel's solution for passing electrons; but the trapped distribution is different. The deeply trapped orbits in our simulation are less populated than in Schamel's solution, which explains why the electron hole in our simulation is narrower at its center. The trapped electron distribution in a steady-state hole is determined by its initialization. We can generate electron holes with different shapes of $f_{\text {trapped }}$ by changing the shape of function $f_{d}$ in our initialization. Our analytic kinematic theory is not affected by the exact structure of an electron hole as it applies to generic electron holes with global charge neutrality.

Hole tracking simulation enables us to visualize directly trapped electrons in a steady state hole. As the simulation domain is moving at the same speed as the electron hole, electrons that are on passing orbits are quickly exchanged out of the domain and only the ones on trapped orbits stay and travel with the solitary wave. Our simulation tracks the step at which a particle is injected. The middle panel of Figure 8 shows the phase-space density of electrons from initialization $f_{e, t=0}$ normalized to current electron phase-space density $f_{e}$ in a steady state hole of potential profile $\phi(x)$. The velocity half-width of densely populated orbits by initial particles in phase-space is around $28 c_{s}$ at hole center, which is approximately equal to $v_{p, e}=\sqrt{\frac{2 e \psi}{m_{e}}}$ for $e \psi=0.23 T_{e}$ as expected. Trapped orbits are therefore almost entirely populated by electrons from initialization. These electrons are trapped from the beginning of the simulation and stay inside the simulation domain with the hole.

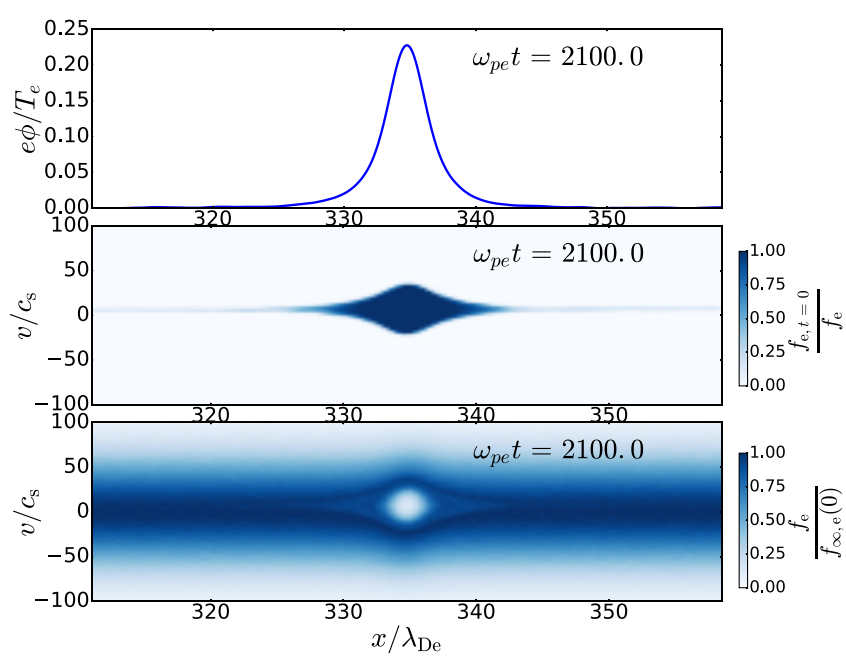

FIG. 8. The hole potential (top panel), the relative phase-space density of initial electrons (middle panel) and the normalized electron phase-space density (bottom panel) at time $\omega_{p e} t=2100$, the hole has a lab frame velocity of $6.9 c_{s}$, $x$ and $v$ are relative to lab frame.
In the run with a relatively high $U_{0}\left(U_{0}>5 c_{s}\right)$, the ions are less perturbed by the initial hole potential as a result of their high relative speed to the hole. This initialization gives a smaller initial transient speed-up as shown in Figure 5. In this case, possible formation of more than one electron hole from our initialization is observed. When this happens, one principal hole is formed which is much deeper than all others. The attraction and coalescence behavior of holes with similar velocity reminiscent of the previous experimental and numerical investigations ${ }^{3,6,27}$ is observed in our simulations. Our hole tracking algorithm tracks the most significant hole signal which is given by the deeper hole. Figure 9 shows a case of two electron holes moving in the same direction with similar speed for a long period of time $\left(\sim 1000 / \omega_{p e}\right)$ until they get close to one another and subsequently their main bulk parts coalesce to one due to mutual attraction. The smaller hole experiences "tidal" deformations ${ }^{27}$ during the interaction and a part of it eventually escapes. A transient increase of magnitude $\sim 0.5 c_{s}$ is observed in the velocity of the principal hole when it interacts with the smaller one. The interaction is relatively smooth because of the disparities between the sizes of two holes.

\section{HOLE PUSHING AND PULLING}

One of the significant advantages of the hole tracking simulation is in which it allows us to study kinematics of an electron hole with slight deviation from its steady state without worrying about losing it from the simulation domain. This feature enables us to probe momentum coupling between ions and an electron hole in quasi steady state.

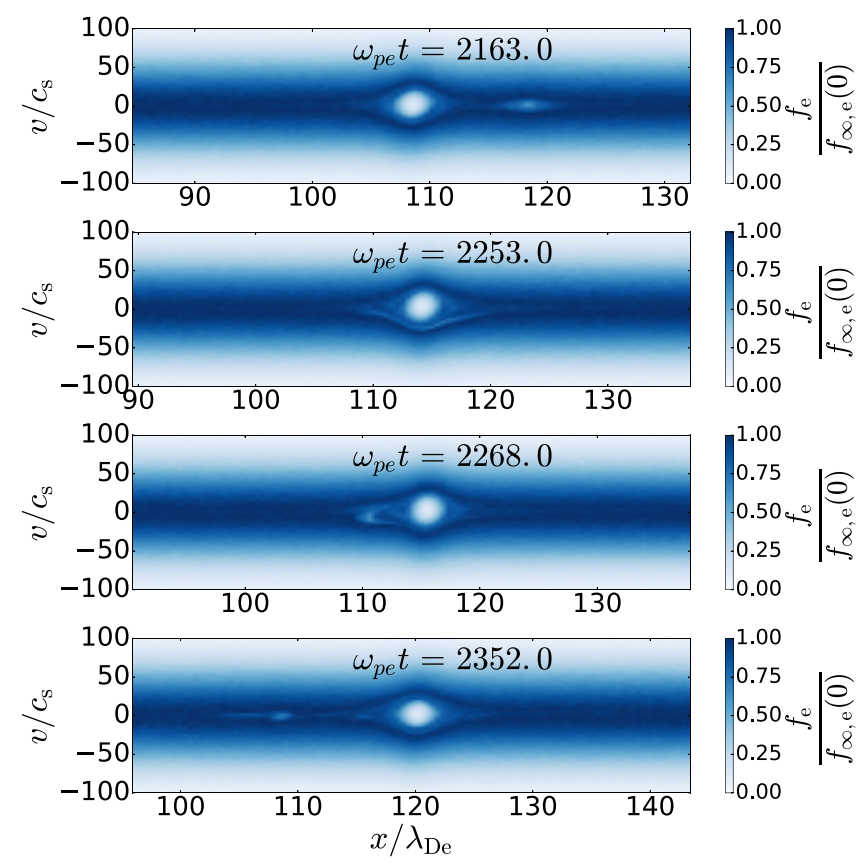

FIG. 9. Coalescence of electron holes of different size in our simulation, a shallow hole is followed by a much deeper one and they eventually partly coalesce. A piece of the shallower hole is sprayed out. This run is performed with $U_{0}=7 c_{s}, m_{i} / m_{e}=1836$ and the deeper hole at the center of simulation domain has a depth of $e \psi=0.23 T_{e}$, the shallower hole has a depth of $e \psi=0.03 T_{e}$ 
In the theory developed by Dupree, the only momentum exchange $^{13}$ between electron hole and ions is through ion reflection by hole potential. This would imply that an electron hole can only "feel" a change in ion momentum when ions begin to reflect from hole potential. For beam-like ions and a shallow electron hole, this only happens when the drift velocity of ions is within several $v_{\text {th }, \mathrm{i}}$ in the rest frame of the electron hole. However, our numerical experiments contradict this conclusion. We clearly see ion influence on electron hole kinematics even when there are effectively zero reflected ions.

The way we investigate this problem is through applying an artificial acceleration to ions. The acceleration is applied by adding an artificial linear background potential to the electrostatic potential that is used to move ions but not electrons in the particle mover of PIC code. The linear artificial potential gives rise to a constant field which accelerates ions at a constant rate. At the same time, ions are injected from a distribution of background ions which is accelerating at the same rate. The overall effect is that all ions experience a constant background field in addition to the field that electrons experience. When ions are accelerated so that their velocity approaches the hole velocity, this is called "pushing." The opposite case is called "pulling." We artificially accelerate ions only after the hole is in steady state. The value of this artificial acceleration is chosen to be on the order of $c_{s} \omega_{p e} / 1000$. The velocity of an ion in the rest frame of a steady state hole is typically a few times sound speed $c_{s}$ in our simulation. The hole spatial width is generally a few times $\lambda_{\text {De }}$ the Debye length. The time it takes for an ion to transit through hole region is therefore on the order of $50 / \omega_{p e}$. During this time, the change in ion velocity due to this artificial background acceleration is around $0.05 c_{s}$, which is much smaller compared to its velocity in hole frame. This choice of artificial acceleration guarantees that short transit-time approximation is valid for ions in these runs.

Figure 10 shows the electron hole velocity evolution in our hole pushing and pulling runs. In the pushing run, constant artificial ion acceleration is applied from $\omega_{p e} t=1500$ to $\omega_{p e} t=3600$ (phase (1)), which accelerates the drift velocity of ions from 0 to $5 c_{s}$ in its initial rest frame. Then acceleration of the same magnitude but opposite sign is applied from $\omega_{p e} t=4500$ until $\omega_{p e} t=6600$ (phase (2)) to bring the ions back to their initial velocity. The pulling run is the same except that we exchange the order of the two acceleration phases (3) (4) instead of (1) (2)). Contrary to what Dupree's theory would predict, the velocity of the electron hole changes instantaneously when the artificial acceleration is applied to ions before ions are reflected by the hole potential. Moreover, the change in hole velocity is always in the same direction as ion acceleration. Results in Figure 10 also show a strong asymmetry in pushing and pulling. For the same initial velocity of the electron hole in ion rest frame, pushing gives larger hole velocity response than pulling. Both pulling and pushing are reversible.

We need the newer theory ${ }^{25}$ of hole momentum conservation to understand the phenomena we see in our simulations. The theory can be generally applied to cases where

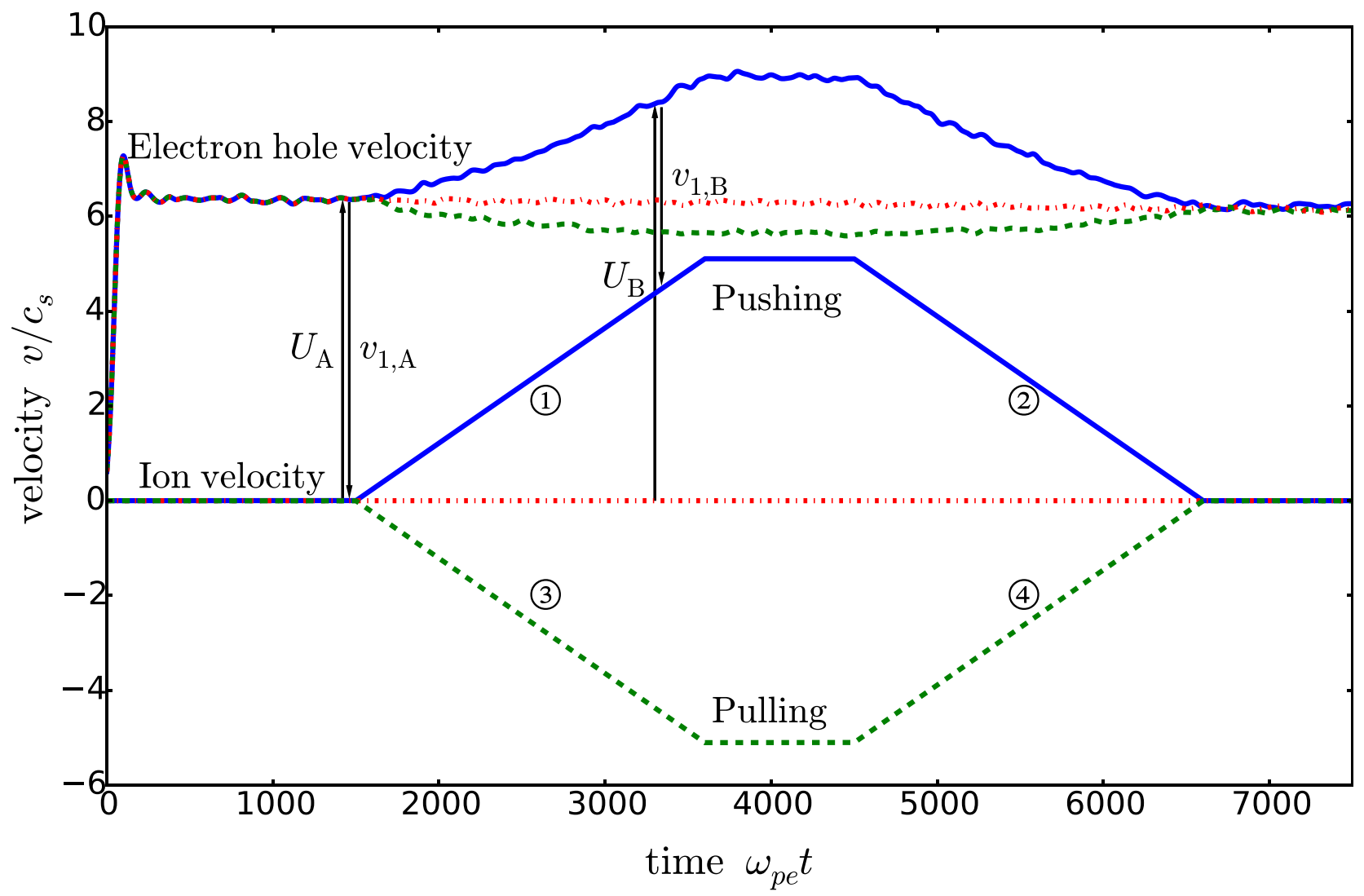

FIG. 10. Hole velocity response to artificial ion acceleration, $T_{e} / T_{i}=20, e \psi=0.1 T_{e}$. Solid line is the "pushing" run and dashed line is the "pulling" run. Dashed dotted line is a reference run where no artificial acceleration is applied. 
one or both species in plasma are subjected to a uniform background force. Hole pushing and pulling can be described by an equation ${ }^{25}$ relating initial and final states A and B during the process of pushing or pulling,

$$
\frac{\Delta U}{c_{s}}=\frac{U_{\mathrm{B}}-U_{\mathrm{A}}}{c_{s}}=\frac{M_{i e}^{4}}{3}\left(\frac{1}{\left|v_{1, \mathrm{~B}} / c_{s}\right|^{3}}-\frac{1}{\left|v_{1, \mathrm{~A}} / c_{s}\right|^{3}}\right),
$$

where $M_{i e}$ is the Mach number of hole velocity at which electron momentum rate magnitude is equal to ion momentum rate magnitude. $M_{i e}^{4} / 3$ is given by

$$
\frac{M_{i e}^{4}}{3}=\frac{m_{i}}{m_{e}}\left[\int_{x_{1}}^{x_{2}}\left(\frac{e \phi(x)}{T_{e}}\right)^{2} d x / \int_{x_{1}}^{x_{2}} h\left(\sqrt{\frac{e \phi(x)}{T_{e}}}\right) d x\right] \text {, }
$$

$\phi(x)$ is the electron hole potential profile with $x_{1}$ and $x_{2}$ denoting its spatial extent (theory assumes that $\phi$ and its derivative vanish at $x_{1}$ and $x_{2}$ ). The special function $h$ is defined by Eq. (9). The definitions of $U_{\mathrm{A}}, U_{\mathrm{B}}, v_{1, \mathrm{~A}}, v_{1, \mathrm{~B}}$ are illustrated by arrows in Figure 10 for a pushing run. $U$ is the velocity of an electron hole in the initial rest frame of ions and $v_{1}$ is the drift velocity of ions in the hole frame.

The theory assumes that the hole does not change its shape during pushing or pulling, so $M_{\mathrm{ie}}$ is a constant for a hole of given potential. Eq. (14) can give us some important insight on how pushing and pulling would behave according to the theory. First, the influence of artificial ion acceleration on hole velocity $U$ is instantaneous. Any finite change in $v_{1}$ will result in change in hole velocity. Second, pushing and pulling are asymmetric. There is no limit on how much a hole can be pushed in its velocity, while pulling is limited by $-M_{i e}^{4} / 3\left|v_{1, \mathrm{~A}} / c_{s}\right|^{3}$. Third, pushing and pulling are independent of path and thus reversible. These properties agree qualitatively with what we see in our simulations.

A quantitative comparison between the theory and our simulations is shown in Figure 11. The theoretical curves of $\Delta U\left(v_{1, \mathrm{~B}}\right)$ shown in solid lines are calculated from Eqs. (14) and (15). The reference velocity $v_{1, \mathrm{~A}}$ is chosen as the velocity of ions in the hole frame right before the artificial acceleration is applied to ions. Its value is determined by the initialization that generates the steady state hole. The calculation of multiplication factor $M_{i e}^{4} / 3$ depends on the exact hole potential profile $\phi(x)$. Since $h(\chi) \rightarrow \chi^{2}$ as $\chi \rightarrow 0,{ }^{25}$ the value of $M_{i e}^{4} / 3$ is $\sim\left(m_{i} / m_{e}\right)\left(e \psi / T_{e}\right)$ for the holes in our simulations which are relatively shallow. We use the potential output from our simulation to calculate a more precise value. The interval $\left[x_{1}, x_{2}\right]$ over which the integrals are performed in Eq. (15) is determined with the electric field. The theory assumes that hole electric field vanishes at its boundaries. Thus, we can practically consider $x_{1}$ and $x_{2}$ to be the two positions where the electric field in our simulation first changes its sign outside the hole center. Once the electric field begins to oscillate on its thermal level, we are in background plasma. The values of integrals in Eq. (15) are evaluated numerically at each time step of acceleration and its average value is used in Eq. (14).

The theory is compared with simulation results in Figure 11 . The simulation velocity is just the hole velocity obtained from the hole tracking algorithm. The hole velocity is filtered by a low-pass filter of cutoff frequency $0.005 \omega_{p e}$. The ions are beam-like and their drift velocity is considered to follow the constant acceleration that is artificially applied. This assumption is adequate as the momentum imparted by ions to electrons is a fraction ${ }^{25}$ smaller than $\sim m_{e} / m_{i}$ of the total momentum injected by the background artificial field.

Numerical experiments show good agreement with the theory. The agreement is further improved by including ion density variation due to hole potential in the theory. This modified solution is plotted in dashed lines in Figure 11. The original theory assumes no ion density perturbation in the derivation of trapped electron density from charge balance. However, when an electron hole is pushed, the relative velocity of ions in the hole frame decreases. The ion density accumulation inside the hole, which is an ion-acoustic soliton attached to the hole in terminology of Saeki and Genma, ${ }^{16}$ becomes more important as ions are slowed down by hole potential. If we account for ion density variation to order $\left(c_{s} / v_{1}\right)^{2}\left(e \phi / T_{e}\right)$, then $h(\chi)$ is replaced by $h(\chi)-$ $\left(c_{s} / v_{1}\right)^{2}\left(e \phi / T_{e}\right)$ in the derivation of Eq. (14). This leads to the modified solution

$$
\left[\frac{U}{c_{s}}\right]_{A}^{B}=M_{i e}^{4}\left[\frac{1}{K^{2}\left(v_{1} / c_{s}\right)}+\frac{1}{2 K^{3}} \ln \left|\frac{v_{1} / c_{s}-K}{v_{1} / c_{s}+K}\right|\right]_{A}^{B},
$$
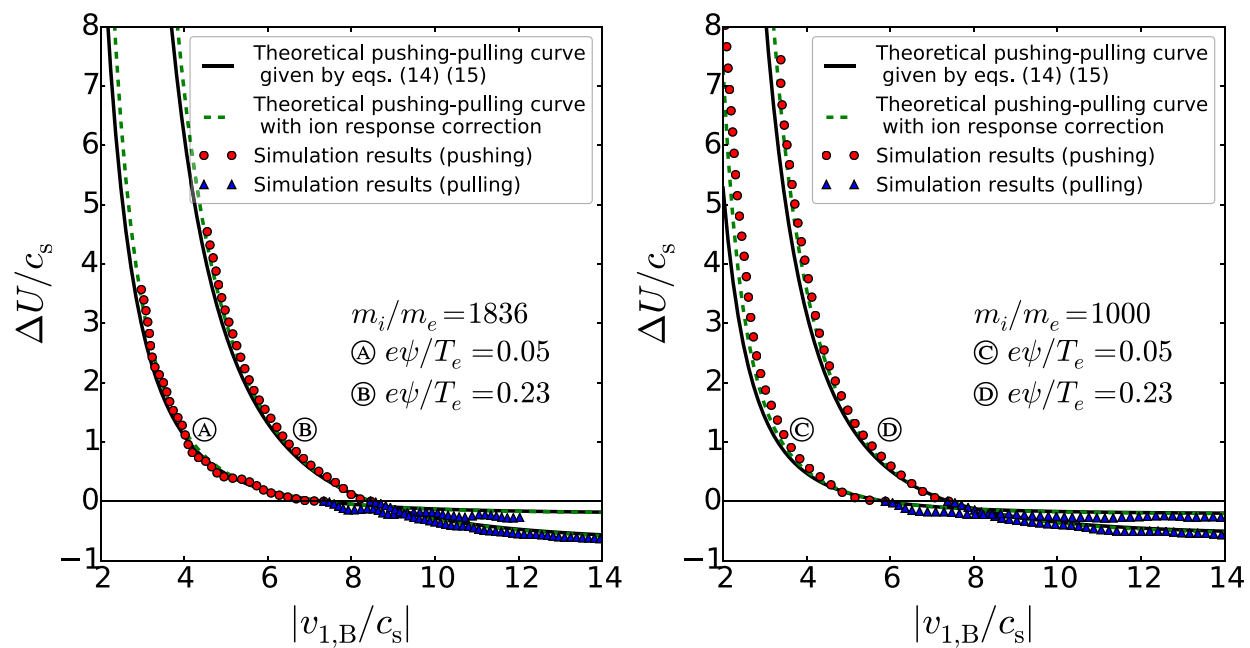

FIG. 11. Hole pushing and pulling runs for holes of different depths using two different mass ratios. The value of $\psi$ is the average value during acceleration. $T_{e} / T_{i}=20, N_{i}=N_{e}=2.56 \times 10^{7}$. 
where $K=\int_{x_{1}}^{x_{2}}\left(e \phi(x) / T_{e}\right) d x / \int_{x_{1}}^{x_{2}} h\left(\sqrt{\left(e \phi(x) / T_{e}\right.}\right) d x$ is a constant. $K$ is evaluated in the same way as $M_{i e}^{4} / 3$ by performing numerical integration over $\left[x_{1}, x_{2}\right]$. For shallow holes, we have $K \simeq 1$ as $e \psi / T_{e} \ll 1$. This correction is more important for small $\left|v_{1}\right|$.

In deriving function $h$, we assume an electron hole situated near the top of the electron distribution. This approximation is no longer adequate when a hole is pushed to an important fraction of $v_{t h, e}$. It is also observed in our simulations that the depth and shape of an electron hole changes slightly $(<5 \%)$ during pushing and pulling. This can have several important implications. The derivation of Eq. (14) assumes no change ${ }^{25}$ in the hole potential. Hole growth and shrinkage will induce hole velocity change as we see in the initial transient. This effect is ignored in our analysis. The change and fluctuations of hole potential in our simulations also make it difficult to evaluate accurately $M_{i e}^{4} / 3$. We observe $5 \%-10 \%$ fluctuations in its value at different time steps. It is the average value that is used in Figure 11.

In hole pushing and pulling experiments, we are trying to resolve small changes in hole velocity to the extent that statistical noise in our simulation needs to be treated carefully. We observe slow spontaneous decay in the velocity of a steadystate hole during long time hole tracking simulation. This effect is shown in Figure 12. The three runs in plot (a) correspond to the pushing-pulling-back run (1) (2), the pullingpushing-back run (3) (4) and the reference run with no artificial ion acceleration presented in Figure 10. Notice the decay in hole velocity even when there is no ion acceleration at all. The change is in diagonal direction for the run with no ion acceleration because $U=-v_{1}$ when ion drift velocity stays constant. The same effect in pushing and pulling runs leads to hysteresis, which can also be seen in plot (a). This slow hole velocity decay is reduced by using more particles in the simulation. The runs shown in the plot (b) of Figure 12 exhibit less velocity decay by virtue of using more particles. Pushingpulling process is also more reversible, which agrees better with our theory predictions. This effect can be explained by detrapping of trapped electrons in an electron hole due to statistical fluctuations in the PIC simulation. The depth of an electron hole $e \psi$ slowly shrinks when the marginally trapped orbits are intermittently connected to background plasma by fluctuating electric fields. Its velocity $U$ decreases as a consequence of its shrinking size. Eq. (8) relates the depth of an electron hole to its velocity. For the run with no ion acceleration shown in plot (a), the hole depth $e \psi$ decreases from $0.1025 T_{e}$ at $\omega_{p e} t=1500$ to $0.098 T_{e}$ at $\omega_{p e} t=7500$. The decrease in hole velocity calculated from Eq. (8) for the amount of depth shrinkage is $\Delta U_{\text {Decay }} \simeq-0.1 c_{s}$. The actual decay in velocity observed in our simulation is $\Delta U_{\text {Decay }}$ $\simeq-0.2 c_{s}$. Though small in magnitude, this effect is important for pulling runs as $\Delta U_{\text {Decay }}$ can be an important fraction of the predicted $\Delta U$. By taking into account this correction, the agreement between our theory and simulation in high $\left|v_{1}\right|$ limit is within $\pm 10 \%$.

Eq. (14) gives no lower limit on how small $\left|v_{1}\right|$ can get by pushing the hole. This is unphysical as ions will be reflected by hole potential if $\left|v_{1}\right|<v_{p, i}$. The modified solution given in Eq. (16) shows that ions cannot approach the hole velocity closer than $\left|v_{1}\right|=K c_{s}$ with $K>1$. In our simulations, we observe that instability occurs before $\left|v_{1}\right|$ can get close to this limit. Ion density is perturbed in the case of instability and the electron hole can be disrupted if pushed further. The exact nature of this instability is beyond the scope of this paper.

\section{SUMMARY}

This paper introduces a new way of simulating a moving electron hole by tracking its motion. The full dynamics of electron hole interacting with ions are studied quantitatively (a)

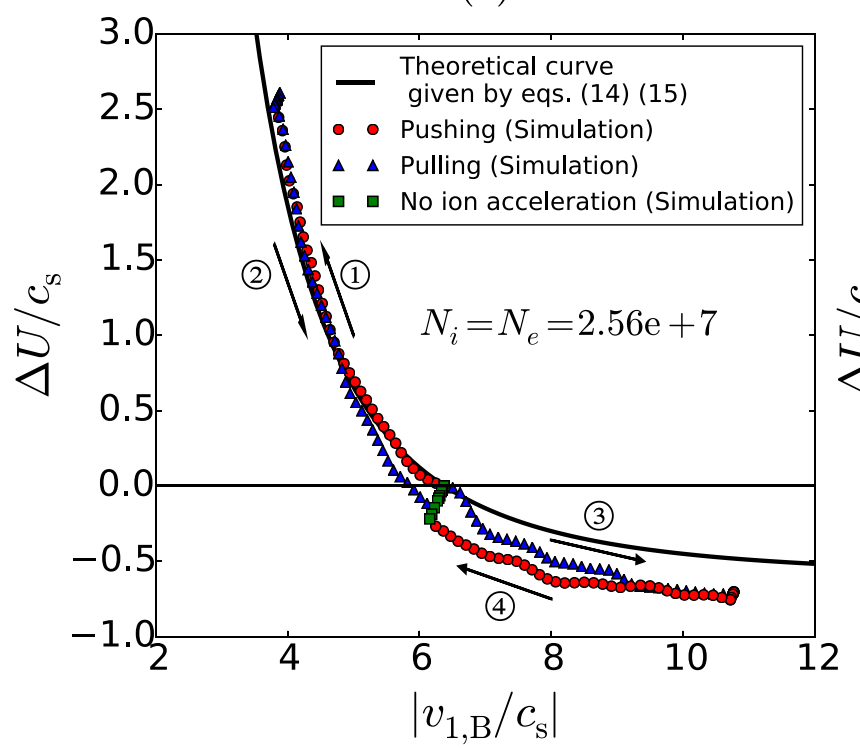

(b)

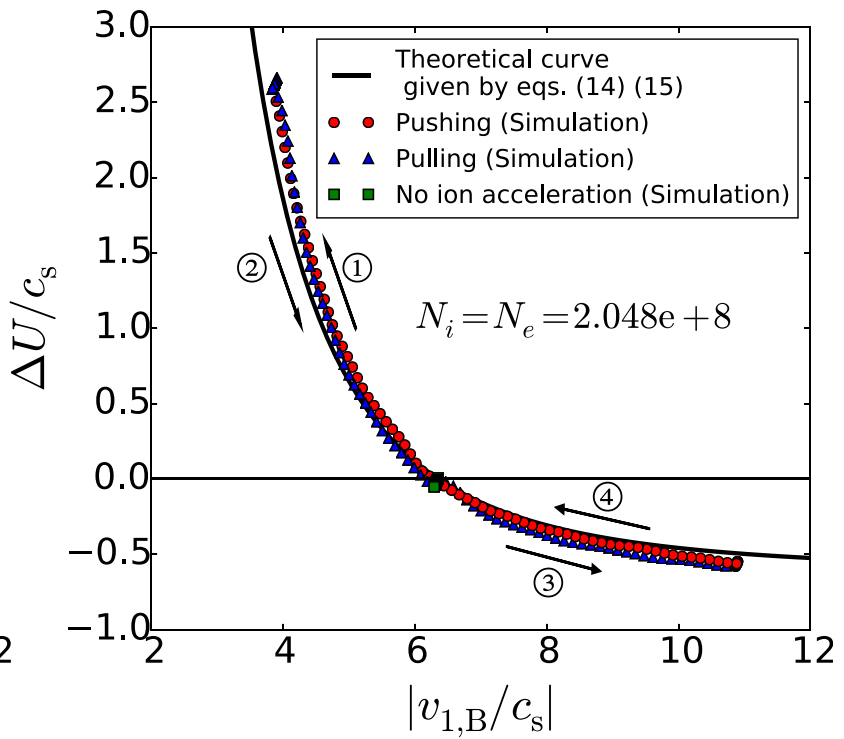

FIG. 12. Illustration of reversibility and hysteresis in pushing and pulling. (a) Pushing, pulling, and no ion acceleration runs showing spontaneous hole velocity decay and hysteresis. (b) The same runs as (a) with eight times as many particles, the spontaneous velocity decay and hysteresis are reduced by using more particles. The number of computation cells is 1000 in these runs and the domain length is $48 \lambda_{\text {De }}$ across. $T_{e} / T_{i}=20, e \psi=0.1 T_{e}$. 
using this computer simulation technique in both transient and steady-state regimes. We find that an electron hole generated using a uniform density initialization approach can be accelerated to several times the ion sound speed by initial ion density perturbation. The final steady-state velocity reached by electron hole depends on ion-to-electron mass ratio, hole depth, and the initial hole velocity in the ion frame. Dynamical coupling of a steady-state hole and ions is investigated by applying a slow artificial acceleration to ions. The velocity of an electron hole reacts instantaneously to changes in ion momentum. An electron hole can be pushed or pulled in velocity by artificially imposed ion acceleration. Pulling and pushing are asymmetric. Simulations are compared with the newly developed analytic theory of hole momentum conservation. Good qualitative and quantitative agreement is presented in this study.

\section{ACKNOWLEDGMENTS}

The hole tracking PIC code used for this work is based on ESPIC developed by Christian Haakonsen. His early input and valuable discussions are gratefully acknowledged. The work was partially supported by the NSF/DOE Basic Plasma Science Partnership under Grant No. DESC0010491. Computer simulations were carried out on the MIT PSFC parallel AMD Opteron/Infiniband cluster Loki.

${ }^{1}$ I. B. Bernstein, J. M. Greene, and M. D. Kruskal, "Exact nonlinear plasma oscillations," Phys. Rev. 108(4), 546-550 (1957).

${ }^{2}$ H. Schamel, "Electron holes, ion holes and double layers," Phys. Rep. 140(3), 161-191 (1986).

${ }^{3}$ K. Saeki, P. Michelsen, H. L. Pécseli, and J. Juul Rasmussen, "Formation and coalescence of electron solitary holes," Phys. Rev. Lett. 42(8), 501-504 (1979).

${ }^{4}$ J. Pickett, L. Chen, S. Kahler, O. Santolík, D. Gurnett, B. Tsurutani, and A. Balogh, "Isolated electrostatic structures observed throughout the cluster orbit: Relationship to magnetic field strength," Ann. Geophys. 22, 2515-2523 (2004).

${ }^{5}$ B. Eliasson and P. K. Shukla, "Dynamics of electron holes in an electronoxygen-ion plasma,” Phys. Rev. Lett. 93(4), 045001 (2004).

${ }^{6}$ J. P. Lynov, P. Michelsen, H. L. Pecseli, and J. Juul Rasmussen, "Interaction between electron holes in a strongly magnetized plasma," Phys. Lett. A 80(1), 23-25 (1980).

${ }^{7}$ J. P. Lynov, P. Michelsen, H. L. Pécseli, J. Juul Rasmussen, and S. H. Sørensen, "Phase-space models of solitary electron holes," Phys. Scr. 31(6), 596-605 (1985).
${ }^{8}$ R. Ergun, C. Carlson, J. McFadden, F. Mozer, L. Muschietti, I. Roth, and R. Strangeway, "Debye-scale plasma structures associated with magnetic-field-aligned electric fields," Phys. Rev. Lett. 81(4), 826-829 (1998).

${ }^{9}$ V. L. Krasovsky, H. Matsumoto, and Y. Omura, "Bernstein-GreeneKruskal analysis of electrostatic solitary waves observed with geotail," J. Geophys. Res. 102(A10), 22131, doi:10.1029/97JA02033 (1997).

${ }^{10}$ L. Muschietti, R. E. Ergun, I. Roth, and C. W. Carlson, "Phase-space electron holes along magnetic field lines," Geophys. Res. Lett. 26(8), 1093-1096, doi:10.1029/1999GL900207 (1999).

${ }^{11}$ J. F. Drake, M. Swisdak, C. Cattell, M. A. Shay, B. N. Rogers, and A. Zeiler, "Formation of electron holes and particle energization during magnetic reconnection," Science 299(1987), 873-877 (2003).

${ }^{12}$ W. Fox, M. Porkolab, J. Egedal, N. Katz, and A. Le, "Observations of electron phase-space holes driven during magnetic reconnection in a laboratory plasma," Phys. Plasmas 19(3), 032118 (2012).

${ }^{13}$ T. H. Dupree, "Growth of phase-space density holes," Phys. Fluids 26(9), 2460 (1983).

${ }^{14}$ J. M. Dawson, "Computer modeling of plasma: Past, present, and future," Phys. Plasmas 2(6), 2189 (1995).

${ }^{15} \mathrm{~K}$. Saeki and J. J. Rasmussen, "Stationary solution of coupled electron hole and ion soliton in a collisionless plasma," J. Phys. Soc. Jpn. 60(3) 735-738 (1991)

${ }^{16} \mathrm{~K}$. Saeki and H. Genma, "Electron-hole disruption due to ion motion and formation of coupled electron hole and ion-acoustic soliton in a plasma," Phys. Rev. Lett. 80(6), 1224-1227 (1998).

${ }^{17}$ I. H. Hutchinson, C. B. Haakonsen, and C. Zhou, "Non-linear plasma wake growth of electron holes," Phys. Plasmas 22(3), 032312 (2015).

${ }^{18}$ C. Bernt Haakonsen, I. H. Hutchinson, and C. Zhou, "Kinetic electron and ion instability of the lunar wake simulated at physical mass ratio," Phys Plasmas 22(3), 032311 (2015).

${ }^{19}$ C. K. Birdsall and A. B. Langdon, Plasma Physics via Computer Simulation (IOP Publishing, Bristol, 1991).

${ }^{20} \mathrm{H}$. Schamel, "Stationary solitary, snoidal and sinusoidal ion acoustic waves," Plasma Phys. 14, 905-924 (1972).

${ }^{21}$ D. M. Malaspina, D. L. Newman, L. B. Willson, K. Goetz, P. J. Kellogg, and K. Kerstin, "Electrostatic solitary waves in the solar wind: Evidence for instability at solar wind current sheets," J. Geophys. Res.: Space Phys. 118(2), 591-599, doi:10.1002/jgra.50102 (2013).

${ }^{22}$ J. E. Gentle, Random Number Generation and Monte Carlo Methods, 2nd ed. (Springer, NY, 2003).

${ }^{23}$ O. Buneman, "Instability, turbulence, and conductivity in current-carrying plasma," Phys. Rev. Lett. 1(1), 8-9 (1958).

${ }^{24}$ T. E. Stringer, "Electrostatic instabilities in current-carrying and counterstreaming plasmas," J. Nucl. Energy, Part C 6(3), 267-279 (1964).

${ }^{25}$ I. H. Hutchinson and C. T. Zhou, "Plasma electron-hole kinematics. I. Momentum conservation," Phys. Plasmas 23, 082101 (2016).

${ }^{26}$ B. D. Fried and S. D. Conte, The Plasma Dispersion Function (Academic Press, New York, 1961).

${ }^{27}$ H. L. Berk, "Phase space hydrodynamics of equivalent nonlinear systems: Experimental and computational observations," Phys. Fluids 13(4), 980 (1970). 